\title{
Cognitive-behavioral therapy for obsessive- compulsive disorder: access to treatment, prediction of long-term outcome with neuroimaging
}

This article was published in the following Dove Press journal:

Psychology Research and Behavior Management

20 July 2015

Number of times this article has been viewed

Joseph O’Neill'

Jamie D Feusner ${ }^{2}$

'Division of Child Psychiatry, ${ }^{2}$ Division of Adult Psychiatry, UCLA Semel Institute for Neuroscience and Human Behavior, Los Angeles, CA, USA
Correspondence: Joseph O’Neill Division of Child Psychiatry, UCLA Semel Institute for Neuroscience, 760 Westwood Plaza \#58-227A, Los Angeles, CA 90024-1759, USA Email joneill@mednet.ucla.edu
Abstract: This article reviews issues related to a major challenge to the field for obsessivecompulsive disorder (OCD): improving access to cognitive-behavioral therapy (CBT). Patientrelated barriers to access include the stigma of OCD and reluctance to take on the demands of CBT. Patient-external factors include the shortage of trained CBT therapists and the high costs of CBT. The second half of the review focuses on one partial, yet plausible aid to improve access - prediction of long-term response to CBT, particularly using neuroimaging methods. Recent pilot data are presented revealing a potential for pretreatment resting-state functional magnetic resonance imaging and magnetic resonance spectroscopy of the brain to forecast OCD symptom severity up to 1 year after completing CBT.

Keywords: follow-up, access to treatment, relapse, resting-state fMRI, magnetic resonance spectroscopy

\section{Introduction}

In the realm of obsessive-compulsive disorder (OCD), expansion of patient access to cognitive-behavioral therapy (CBT) is a major clinical challenge. The first half of this review summarizes this problem and some attempts at solution. We mostly comment on the situation in the US, especially California, although similar conditions exist elsewhere. The second half of the paper focuses on one aspect of CBT - post-treatment follow-up. Effective prediction of long-term (or even short-term) response to CBT or other OCD treatments is lacking. Improved prediction would partially alleviate the access issue by enabling better allocation of scarce therapist and financial resources and by increasing confidence of patients, prescribers, and third-party payers in CBT.

\section{Factors limiting access to CBT for OCD}

CBT, particularly intensive daily $\mathrm{CBT},{ }^{1,2}$ is highly effective for $\mathrm{OCD}^{3}$ with response rates of $50 \%-70 \%{ }^{4,5} \mathrm{CBT}$ offers comparable symptom improvement and lower relapse than time-limited pharmacotherapy. ${ }^{6-12}$ In contrast to serotonin-reuptake inhibitors (SRIs) or other drugs, moreover, CBT has faster initial response; a response that typically persists past the end of treatment (ideally, for life); and no physical untoward "side-effects". Finally, through CBT, a patient acquires self-mastery, insights, and life skills not imparted by medication. Thus, CBT is established as a first-line, evidencebased treatment for OCD. ${ }^{13}$

OCD treatment generally is underutilized ${ }^{14}$ and, compared with SRIs, CBT is vastly underused. Few OCD patients actually receive CBT: ${ }^{15-17}$ only $\sim 5 \%$ of adult patients in British and American surveys. ${ }^{18,19}$ This is unfortunate because there are 
substantial numbers of OCD patients who respond to CBT or to combined CBT and medications who do not respond to medications alone. That is, there are patients who will not get better if they do not receive CBT. At present, there is no way to tell a priori if a patient will be a responder or a non-responder. There is also a subgroup of patients who might benefit from medications but who refuse to take them, leaving CBT as the only major treatment option. Thus, many patients who could benefit from CBT are not receiving it.

\section{Patient-related factors limiting access}

Numerous sufferers of OCD, wittingly, willingly, or not, deny themselves access to CBT. The full range of insight into one's own condition is represented in OCD; thus, some patients have low insight. ${ }^{20}$ It may not occur to such individuals to seek treatment without considerable prodding from commiserating friends and family. Many other individuals are all too aware or at least suspicious that something is wrong, but are powerfully dissuaded from seeking treatment (even led to denial) by the stigma of OCD. As for mental illness generally, this stigma has abated in recent decades, but still very much exists. Other sufferers are not aware of CBT as a treatment option. Yet others are, to varying degrees, aware but doubtful of the efficacy of CBT. They perhaps may be categorically dismissive of psychotherapy "talking cures". A further group may have a more or less concrete grasp of what CBT entails and eschew it for that every reason. That is, they are reluctant to take on the work and stress of CBT, in particular the exposure to and confrontation with one's deepest fears. ${ }^{21}$ Such attitudes may constitute one form of insufficient "motivation". Others may be insufficiently motivated because they feel their OCD is not severe or life-disrupting enough to merit treatment. All these factors in the minds of prospective patients can militate in varying combinations to forestall reaching out to get badly needed help.

A second class of prospective patients are disqualified from or poorly suited for CBT based on specific psychiatric comorbidities. If a patient's depression is too severe, then it is likely he or she will not muster the energy or have the motivation required to practice CBT exercises. Hence, prognosis is poor and many therapists choose not to work with such patients until their depression is better managed with medications and/or psychotherapy for depression. The same applies for bipolar patients currently in the depressive pole. Bipolar patients experiencing mania or hypomania, in contrast, are poor candidates for CBT because they are feeling too good! Hence, they are unlikely to experience the fear and anxiety generated during CBT exposure exercises, emotions thought to drive recovery. In a similar vein, patients with currently active substance abuse are poor candidates for CBT. For them, the effects of the substances may interfere with their ability to experience the requisite anxiety or distress necessary for success with exposure exercises. Comorbid psychoses can also preclude treatment with CBT. Those with severe personality disorders or otherwise severe characterological disturbances are generally poor candidates for OCD-focused CBT. These comorbidities temporarily or permanently exclude a portion of the psychiatric population from CBT.

\section{Patient-external factors limiting access}

One main reason for the underuse of CBT is the lack of highly trained CBT therapists in the health care system. ${ }^{22-25}$ Many OCD patients who desire CBT cannot get in to see a qualified therapist within a reasonable timeframe. Most graduate programs in psychology and allied fields include only minimal training in CBT and no training in exposure and response prevention (the best-established form of CBT) in their standard curricula. Most therapists are currently trained by senior colleagues or learn on their own at their practices. A consequence of the shortage of therapists is that the clinics that do offer CBT services often maintain long waitlists. Therefore, many patients who would benefit from CBT are not receiving it, or only after a long delay.

Another limiting factor is the cost of CBT. CBT is chiefly administered in "weekly" or "intensive daily" modalities. In the former, the patient undergoes one 1-2-hour session every week for 12-14 weeks; in the latter, the patient undergoes treatment for 4-5 hours, 4-5 days per week for 3-6 weeks. For both modalities, the patient is also required to perform "homework" exercises outside the sessions. Either way, there are heavy demands on the patient's and the therapist's time. As the therapist is normally a highly trained professional, substantial fees can incur. This is in addition to costs to the patient in time away from work, school, raising children, and other life activities. Some patients judge these costs to be unsustainable and opt for drug treatment instead, even if in the long run they end-up spending more on medication. Thus, cost can be a substantial barrier to CBT.

The following discussion of CBT and health insurance is most relevant for US practitioners. But it does have some relevance for clinicians elsewhere, for example, in countries where prescription of more than a standard amount of CBT (eg, ten sessions) has to be justified to a public health agency. 
Health insurance can mitigate the costs of CBT. Many patients prefer to pay for $\mathrm{CBT}$ through their insurance, especially since they are paying premiums for it, directly or indirectly through payroll deduction. But not all patients have health insurance, or enough insurance, or the right kind of insurance. Generally, in the US, if a patient has a preferred provider organization type health insurance plan, there will be coverage for CBT, although it may only pay for $1 / 3$ to $2 / 3$ of the costs. For more economical plans, that may not be the case. Some patients are not aware that insurance even covers $\mathrm{CBT}$. Access to the Internet has ameliorated this problem recently, but it is still challenging and confusing to determine exactly what is covered and what not. Insurance reimbursement for CBT is not universal and can be inadequate. It also varies across policies and is often opaque and byzantine in complexity, which often poses too difficult a task for someone with OCD who may be easily overwhelmed. It is typically easier to obtain coverage for prescription drugs than for CBT. Some patients with insurance cannot afford even the residual costs (deductibles, co-pays, etc) after insurance, although therapists may offer discounts in such cases. For patients seeking "in-network" coverage, insurance companies will provide them a list of therapists who self-report that they use CBT treatment modalities. However, there is no universal credential for CBT aptitude, and many patients find that these therapists have minimal knowledge of, much less expertise with, CBT. Still other patients do not wish to pay for CBT through insurance even if they can, for fear of compromising privacy and other reasons. Many therapists do not accept insurance due to the complexities and limitations and because reimbursement rates are too low. Even when claims are awarded, payment may be protracted. Small private practices are especially impacted by the bureaucratic hindrances associated with third-party payers. While large hospitals may maintain entire departments (eg, "utilization review") tasked with justifying proposed treatments to third-party payers and collecting reimbursement therefrom, fewer private therapists have the time and resources for such activity. This is a major reason why many small practices do not accept insurance. After all, for hourly professionals, their time is their livelihood. Even in large centers, many clinical staff can spend nearly as much time filling out forms or on the phone with insurance companies trying to establish "medical necessity" as they do actually treating patients, a deplorable state-of-affairs. Insurance providers argue that claim-resistant practices actually benefit the patient population by way of "cost containment", but costs could also be contained by accepting lower profits and/or improving efficiency, without denying needed services to patients. In sum, insurance renders CBT accessible for many patients, but substantial issues remain.

\section{Responses of the field to CBT access issues}

Certain patient-related barriers to access, including lack of insight, stigma of OCD, and awareness of CBT and its efficacy, can be approached in part by the methods of public relations. The International Obsessive-Compulsive Disorder Foundation (IOCDF, http://iocdf.org/) has done tremendous work in these areas over the years. The advent of the Internet has also helped reduce the stigma of OCD and has allowed many patients to gain anonymous access to sensitive information about OCD and its remediation. Perhaps ongoing public relations work could be supplemented by specialized outreach efforts targeting patients who are frightened of embarking on a regimen of CBT. The latter could be particularly effective if patients who have successfully undergone CBT treatment could testify to its benefits, and encourage other prospective patients.

In the case of patients insufficiently motivated to undergo CBT, often when the disease has grown to unmanageable severity and one or more other forms of treatment have failed, some patients will finally opt for CBT. One would, of course, rather that they not wait that long. This has driven efforts to propose motivational therapy before a regimen of CBT for appropriate patients. Similarly, for patients with depression, bipolar disorder, substance abuse, and/or psychoses, these conditions can be treated first with medication and/or psychotherapy before inaugurating CBT for OCD.

The issue of the shortage of trained CBT therapists is being countered in part by the IOCDF, which offers CBT training for licensed therapists by top leaders in the field through their Behavior Therapy Training Institute (BTTI) http://iocdf.org/professionals/training-institute/btti/ three times per year at relatively modest cost due to partial subsidization through the foundation. However, many more expert therapists are needed than are generated by the BTTI alone. It would be helpful if training in exposure and response prevention was mandatory for graduate programs in clinical psychology and allied disciplines, at least in some schools.

Issues involving patients having insurance coverage for CBT, insufficient reimbursement of CBT costs, limits on the amount of CBT covered, bureaucratic burden in securing coverage and reimbursement are traditionally addressed (adequately or not) in the arena of public health policy through litigation (eg, class-action lawsuits), lobbying, and 
political activism. Part of this is subsumed under efforts to improve access to mental health services, generally. Past successes have included the passage of mental health parity laws in the US and California requiring that third-party benefits for mental health be no lower than those for medical conditions. Unfortunately, through technicalities, third-party payers have been able to sidestep the spirit of these laws. Similarly, the enactment of the Affordable Care Act in the US has not solved all issues related to cost and insurance, although it has enabled some OCD patients to gain access to CBT because they can no longer be denied coverage on grounds of pre-existing condition. Given the overwhelming financial and political power of interests vested in minimizing and reducing outlays for health insurance claims, it would likely require substantially higher sustained activism by patients and professionals and their organizations to bring about the needed reforms. They would thereby need to remain vigilant of perils of compromise and co-optation.

A perhaps more promising innovative response of the field to combined cost and therapist-availability issues has been to investigate alternative modalities of CBT that are less time intensive for the therapist. Group - as opposed to traditional individual - CBT has recently been studied intensively. ${ }^{26-30}$ These group CBT studies are reporting efficacies that are sometimes slightly less, but generally comparable to responses to individual CBT and to SRIs. Telephone- or Internet-based CBT has also been explored in multiple studies in adult and pediatric OCD. ${ }^{31-36}$ These studies, again, report efficacy comparable to traditional treatment modalities. Such approaches appear to offer particularly practical solutions to the challenges of cost and therapist availability.

\section{Prediction of post-CBT outcomes in OCD}

The remainder of this review concerns a circumscribed aspect of the issue of access to CBT: prediction of post-treatment outcome. Effective prediction of long-term (or even immediate) outcome does not exist for any OCD treatment, including CBT. If developed, then such would alleviate patient-related and patient-external barriers to CBT. In particular, if we knew better in advance who will respond (especially long-term) to CBT and who will not, it would enable more efficient prescription of CBT and superior allocation of scarce therapist resources. Moreover, a clinician could advise an individual patient of the odds of success of CBT before undergoing therapy and/or odds of relapse after therapy. Patients with poor odds could be referred to other forms of treatment.
Although every patient with OCD - including those for whom poor response is foreseen - should be allowed to try CBT if he or she so prefers. But those with good prospects could be more strongly encouraged to try CBT, even against initial reluctance. If we had post-treatment indicators whether relapse is likely or whether benefits achieved will probably be maintained, we would be able to lengthen CBT or to divert to an alternative therapy (and justify these choices to third-party payers). In either case, clinicians, including psychiatrists and general practitioners, would be more inclined to prescribe CBT alongside or instead of medications to those patients with good prospects.

\section{Relapse after CBT for OCD}

As the effectiveness of CBT is well documented and beyond doubt, a more useful clinical question is not who will respond to treatment but rather who will remain well and/or continue to improve after treatment. Although, as mentioned, relapse is less common after CBT for OCD than after discontinuation of medication, post-treatment relapse still occurs in approximately $20 \%$ of CBT patients. ${ }^{37-43}$ Across studies, relapse rates range widely from $0 \%$ to $50 \%,{ }^{7}$ but relapse can occur even when an explicit relapse-prevention component is included in the CBT regimen. Wide variability in relapse may relate to differences in treatment parameters, patient characteristics, and even the definition of relapse. ${ }^{12}$ Given the substantial time commitment of CBT, its expense, the high demand yet shortage of qualified therapists, and consequent long waiting lists, relapse represents an unanticipated yet untoward misallocation of resources and a heavy burden on patients, clinicians, and public health. Sadly, the factors that predict who will retain improvement and who will relapse are unknown. Thus, the question is significant: What predicts who will remain in remission and who will relapse after treatment? Moreover, adequate studies have not been done to determine the duration of treatment optimal for preventing relapse. ${ }^{44}$

Despite the clinical importance of determining to what extent worsening of OCD after treatment should be considered "relapse", remarkably little research has addressed this topic. Several treatment studies have investigated relapse, using a range of definitions. These include $50 \%$ or greater worsening of Yale-Brown Obsessive Compulsive Scale (Y-BOCS), Y-BOCS $\geq 19$, or a Clinical Global ImpressionImprovement (CGI-I) score of "much worse" or "very much worse"; ${ }^{10}$ worsening of $\geq 5$ points on Y-BOCS, Y-BOCS of $\geq 20$, and at least a 1 -point increase on CGI-I; ${ }^{45}$ return to pretreatment Y-BOCS or $\mathrm{a} \geq 1$-point worsening on CGI-I; ${ }^{46}$ $\geq 5$-point worsening on Y-BOCS or investigator's judgment of 
unsatisfactory treatment effect; ${ }^{47,48}$ or return to pretreatment severity on the CGI-Severity (CGI-S) scale. ${ }^{11,49}$ It would be beneficial for the field to reach a uniform standard definition of relapse.

\section{Clinical predictors of relapse}

While there is some evidence for predictors of short-term response to treatment, ${ }^{50-52}$ fewer studies have addressed the equally important question of sustained response, or, conversely, worsening after treatment. ${ }^{53,54}$ Several naturalistic follow-up studies have sought to identify predictors of symptom course..$^{29,55-60}$ One study followed a cohort for 21 months after a 12-week randomized trial of group CBT or fluoxetine. ${ }^{58}$ Comorbid affective disorders, any comorbid psychiatric condition, and illness duration were associated with higher post-treatment OCD symptoms. A study that followed an OCD cohort treated with 12 sessions of group CBT for 2 years found that full remission of symptoms at the end of treatment protected against relapse. ${ }^{29}$ Another study followed patients for 6 years after receiving CBT with clomipramine or placebo. Greater amount of CBT, better homework compliance, and improvement at the end of treatment were associated with better outcomes. ${ }^{44}$ Likewise, in a meta-analysis of pediatric OCD, severity of illness, comorbid diagnoses, and poor initial treatment response were associated with worse longitudinal course ${ }^{60}$ However, another study that followed patients 6-8 years after CBT plus fluvoxamine or placebo did not find an association between short-term treatment response and long-term outcome. ${ }^{59}$ In aggregate, naturalistic studies have examined group or individual CBT, alone or combined with pharmacotherapy, over follow-up periods ranging from 2 to 30 years. Predictors of long-term success varied widely and included shorter duration of OCD, lower severity, lack of psychiatric comorbidities, more CBT, and better CBT homework compliance. Across studies, the most consistent finding is that shorter illness duration predicts better outcome. While this counsels early intervention, it cannot be the sole factor, as response to $\mathrm{CBT}$ occurs in many long-term untreated OCD patients and not in every short-term patient. A few studies pointed to full remission as a predictor of continued post-treatment well-being. While remission is increasingly aspired to as a treatment goal, ${ }^{61}$ complete remission remains rare in adult OCD. Moreover, many demographic factors, and even duration of illness, may be useful prognostically but are not modifiable, reducing their clinical utility. For example, the individual who is seeking treatment but has already had the illness for many years cannot "turn back the clock". In principle, predictors based on psychometric instruments (or neurocognitive profiles) may be less reliable than objective measures (if such can be identified) as the former are highly effort- and, in some cases, evaluator-dependent. Thus, none of the above diverse endpoints has been adopted as a reliable clinical predictor. Moreover, the above results say little about brain mechanisms of relapse prevention.

\section{Brain-based predictors of relapse}

Prognostic biomarkers of relapse, in contrast, could lend more objectivity to clinical decision-making, while informing on possible brain mechanisms. Pretreatment signs of relapse, for example, could help determine whether an alternative treatment should be pursued, while post-treatment signs could help clinicians decide whether additional (or different) treatment is warranted. This would help alleviate the issue of the shortage of qualified CBT therapists since available therapists could be assigned to cases with higher prospects of success. Patients with low prospects on CBT could be treated with medication or other therapies. By increasing the long-term efficacy of CBT, an accurate biomarker test might even encourage third-party providers to cover CBT more readily, thus increasing access for patients who cannot afford it. Ie, an insurance company or a public health care agency with power to restrict or deny payment for or access to CBT sessions will more likely view such sessions as a "good investment" and therefore authorize a course of CBT if objective biomarker signs point to significant positive response (especially long-term) for an individual patient. Candidate biomarkers include safe, non-invasive, in vivo electroencephalography or magnetic resonance imaging (MRI)-based measures of brain-electric activity, structural morphometry or connectivity, functional connectivity, or neurochemical profiles. If such endpoints can be associated with relapse prior to treatment, then they could help determine if alternative therapy should be pursued.

Neuroimaging measurements of brain functional and metabolic activity are logical targets for putative biomarkers of clinical outcome. (For readers unfamiliar with neuroimaging, we recommend a review ${ }^{62}$ aimed at non-imaging clinicians.) Multiple studies associate OCD with regional hyperactivity, particularly in the familiar frontostriatal OCD brain circuits. ${ }^{63,64}$ One popular neuroimaging modality used to measure functional activity is resting-state functional MRI (rsfMRI) based on the blood oxygen level-dependent (BOLD) effect. The BOLD effect measures the oxygenation of hemoglobin in the blood in the tissue scanned. Neurons fire more when active, consume more oxygen when they fire, and extract this oxygen from the blood, changing its 
paramagnetic properties. This results in a BOLD signal within the magnetic field. Therefore, a stronger BOLD effect in a given volume of brain tissue suggests higher neuronal activity in that volume. fMRI divides the brain into a $3 \mathrm{D}$ grid of thousands of small (usually $2-3 \mathrm{~mm}^{3}$ ) blocks, called volume elements or "voxels", and measures the BOLD effect in each. With "task" fMRI, usually the patient is performing an assigned task, eg, viewing a stimulus or performing a neurocognitive test. In this way, task fMRI detects which parts of the brain are active during each specific task. rsfMRI is collected when there is no task; rather, the patient lies quietly in the scanner but does not go to sleep. It turns out the brain is still quite active in this state. In rsfMRI analysis, one calculates the correlation coefficients between the BOLD activities of each pair of voxels across the runtime of the scan as a measure of 'functional connectivity'. Alternatively, one can calculate across anatomically- or functionally-defined regions. Such analyses have revealed that the brain is divided into a number of functional networks of interconnected anatomic regions. Many of these networks that show high connectivity at rest are the same as those that activate during specific tasks. This has been demonstrated in that performance of these tasks outside the scanner typically correlates with the intrinsic resting-state connectivity of the network involved in processing this task. ${ }^{65}$ Why are these networks active at rest? It may be due to the fact that there are spontaneous low-frequency fluctuations in these networks - even though they are not in use, every so often they spontaneously fire together (neurons that "fire together wire together"). ${ }^{66}$ Moreover, as OCD patients live very much "in their heads", overthink, are subjected to intrusive obsessive thoughts, etc, it is plausible that they could also, or alternatively, exhibit abnormal rsfMRI activity due to the abnormal activity in the scanner. Indeed, fMRI studies have detected aberrant network connectivity in frontostriatal and parietal regions that may contribute to OCD symptoms. ${ }^{67,68}$ Below we report on our research involving rsfMRI and longterm post-CBT outcome in OCD.

Another imaging modality, proton magnetic resonance spectroscopy (MRS), has also been applied to OCD. ${ }^{69}$ In MRS, one or more (usually larger, maybe 3-4 cc) voxels in targeted brain regions are stimulated with a (brief, nonharmful) pulse of radiofrequency radiation from the scanner headcoil. This pulse is absorbed by the hydrogen nuclei (protons) in the various biomolecules ("metabolites") in the tissue, which promotes the protons into an energetically excited state. Over 10 s to 100 s of milliseconds, these protons then relax back to their initial unexcited state. As they do this, they release the energy they had absorbed. The liberated energy is picked up by the coil and registered as an electrical voltage (signal) read by the scanner. The key to MRS is that the protons in each different metabolite absorb and then release energy at a slightly different frequency. Thus, the mix of frequencies in the coil signal reveals which species of metabolites are present in the tissue. The "MR spectrum" is a plot of the signal as a function of the frequency and consists of a series of "peaks". The location of each peak on the frequency axis tells which metabolite the signal arises from. The size of the peak is proportional to the concentration of the metabolite in the voxel. It turns out that, of the thousands of biomolecules in the brain, only 5-6 are easily measured with conventional clinical MRS, ie, at scanner field strengths of 1.5-3 Tesla (T). Fortunately, these 5-6 are physiologically significant metabolites, in particular, ones related to brain-energy metabolism. From positron emission tomography studies, it is known that specific brain regions are energetically hyperactive in OCD, and this hyperactivity reduces shortly after treatment, including CBT (reviewed in O'Neill et $\mathrm{al}^{70}$ and Saxena et $\mathrm{al}^{71,72}$ ). It is thus reasonable to anticipate that MRS metabolites in these regions are sensitive to OCD and/or its treatment. Indeed, in prior work, we $\mathrm{e}^{70,73}$ and others (reviewed in Brennan et $\mathrm{al}^{69}$ ) found differences in levels of MRS-detectable metabolites between OCD patients and healthy controls, as well as changes in metabolite levels immediately following OCD treatment, including CBT. Below we report on our research involving MRS and longerterm post-CBT outcome in OCD.

Both rsfMRI and MRS are readily performed on standard clinical MRI scanners. MRS can sample most single brain regions at a resolution of 3-4 cc in 5-10 minutes. In the same time span, rsfMRI can sample the entire brain at a resolution of 2-3 $\mathrm{mm}$. With some effort, these procedures can be replicated across different scanners, lending them for use in large, multisite clinical trials. If rsfMRI and/or MRS endpoints reliably predicting short- and/or long-term response to $\mathrm{CBT}$ can be identified, then these properties will ultimately suit them for actual use in clinical settings. Reliable findings could also set the stage for testing different treatment durations to reduce risk of relapse.

\section{A pilot rsfMRI correlate of post-CBT outcome}

To give an idea of the flavor and potential of neuroimaging to predict CBT outcomes, in this section we present some of our recently published rsfMRI data. ${ }^{74}$ In the next section, we present original MRS data from the same pilot study. Data 
from both modalities were acquired in a larger investigation of intensive outpatient CBT for adult OCD in a subcohort of 17-19 patients (aged 21-50) examined in post-treatment follow-up. These patients were reevaluated with multiple clinical instruments at times ranging 1-12 months (mean

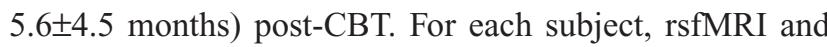
MRS were co-acquired within 1 week prior to initiating treatment and again within 1 week after finishing treatment. Scans were performed in a 3-T Siemens Trio scanner using a 12-channel phased-array headcoil.

Whole-brain BOLD rsfMRI was acquired using an 8-minute echo-planar imaging sequence (repetition time/ echo time $=2,000 / 25$ milliseconds, flip angle $=78^{\circ}$, voxels $3 \times 3 \times 3 \mathrm{~mm}^{3}, 1 \mathrm{~mm}$ gap). Subjects were instructed to rest with eyes closed, to remain as still as possible, and not to sleep. rsfMRI data were analyzed with graph theory. Graph theory has recently yielded impressive results in the analysis of brain connectivity networks. ${ }^{75}$ In graph theory, the fMRI dataset is represented abstractly as a brainwide geometric network or "graph" of (in our case 160) anatomical "nodes" or network vertices. A node consists of a clump of contiguous fMRI voxels all in the same predefined brain region. A single "edge" is drawn on the graph between each pair of nodes that have above-threshold functional connectivity (ie, internode BOLD activity correlation coefficient), after partialing-out noise contributions. One then calculates various parameters that characterize the shape or "topology" of the resulting graph and, if successful, the organization of the underlying brain network. These parameters answer questions such as: Do nodes on average have many connections or few connections? Are nodes mostly connected with their near neighbors or with other distant nodes? The most interesting graph theory parameter in our findings was "small-worldness". Like a "hub and spoke" city subway system that overlays local lines making frequent stops with express trains having fewer stops, a network with small-world architecture features dense local connections alongside sparser remote connections between nodes. This pattern is highly efficient for information transfer in the brain. ${ }^{76}$

In our pilot sample, we found that group-mean smallworldness rose a modest but significant amount after CBT (7.5\%,P<0.05, with permutation tests, controlling for motion; Figure 1). Small-worldness increased in 16 of 17 patients (though in 2 cases only minimally). Patients who already had high pre-CBT small-worldness showed the least increase. Moreover, such patients were most likely to experience worsening of OCD symptoms at follow-up (Figure 1). The six patients who qualified as post-CBT relapsers ( $\geq 5$ points increase in Y-BOCS total score relative to score at discharge) had above-average pre-CBT small-worldness. There have been few prior graph theory studies of OCD, but one ${ }^{77}$ revealed below-normal small-world architecture in untreated OCD patients. A second study found that small-worldness increased in OCD after SRI medication. ${ }^{78}$ Combining these results with ours suggests that at least some OCD patients have below-normal efficiency of brain information processing. Raising this efficiency may be one CBT- or drug-mediated path to recovery from OCD (or, alternatively, a side benefit of recovery). But OCD patients who already have efficient brains may be more liable to relapse. One highly speculative explanation of this observation is that OCD "hi-jacks" a patient's mental faculties as part of symptom fulmination. More efficient cognitive faculties plague some OCD patients who find themselves misusing these
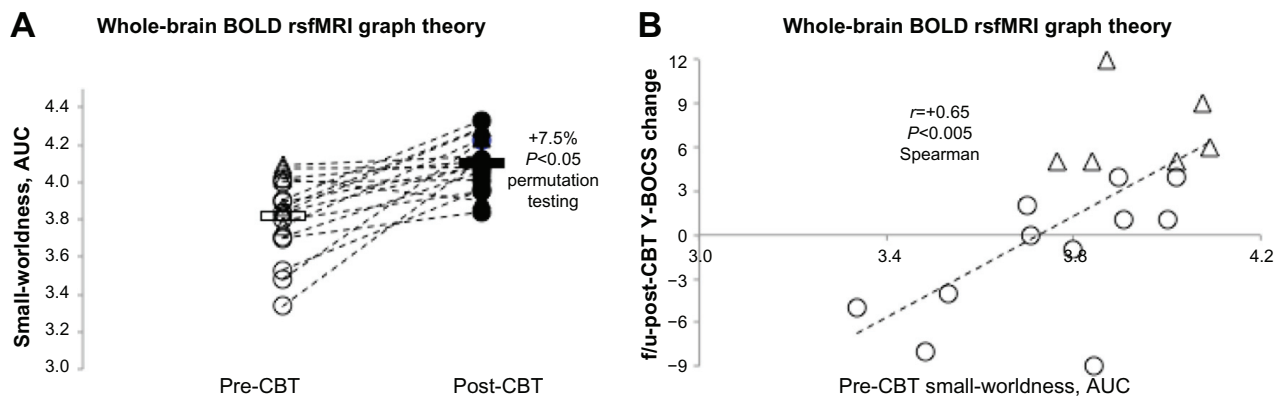

Figure I A pretreatment brain imaging predictor of long-term treatment response of OCD.

Notes: (A) Values of the resting-state fMRI (rsfMRI) graph theory parameter small-worldness (a metric of brain information transfer efficiency) in a pilot sample of I7 adult OCD patients within I week before (pre; open symbols) and I week after (post; filled symbols), 4 weeks of intensive daily CBT. Sixteen of I7 patients (2 minimally) exhibited pre-to-post increase in small-worldness (group-mean 7.5\%, $P<0.05$ permutation testing). (B) For same sample, change in $Y$-BOCS total score measuring severity of core OCD symptoms, expressed as score at post-CBT follow-up minus score immediately after completing CBT. This is plotted as a function of pre-CBT small-worldness. Patients with higher pre-CBT small-worldness had worsening of OCD symptoms (positive change) at follow-up while patients with initially low small-worldness had improvement in symptoms (negative change) $(r=0.65, P<0.005)$. Relapsers ( $\geq 5$-point increase in $Y$-BOCS; triangles) had above-average pre-CBT small-worldness. Thus, rsfMRI graph theory may have some ability to predict long-term treatment response in $O C D$.

Abbreviations: AUC, area under the curve; BOLD, blood oxygen level-dependent; $\mathrm{OCD}$, obsessive-compulsive disorder; rsfMRI, resting-state functional magnetic resonance imaging; fMRI, functional magnetic resonance imaging; CBT, cognitive-behavioral therapy; Y-BOCS, Yale-Brown Obsessive-Compulsive Scale. 
faculties to conceive of more numerous and sophisticated obsessions, rituals, and avoidance strategies, all of which incline to relapse. Another possibility is that for CBT to have enduring effects, there needs to be a reorganization of networks in the direction of increased efficiency. Those with high small-worldness are already at their "ceiling" and thus further reorganization cannot occur. Although they may experience diminished OCD symptoms from pre- to post-treatment, they cannot maintain this new state because this change was not contemporaneous with a reorganization of the network. In any case, these findings hint at a possible ability of rsfMRI graph theory to discriminate different subtypes of patients in terms of post-CBT response.

\section{A pilot MRS correlate of post-CBT outcome}

MRS was acquired from the midline (ie, average of left and right hemispheres) pregenual anterior cingulate cortex (pACC). On sagittal brain MRI, the pACC lies just anterior to the corpus callosum (Figure 2). Water-suppressed MRS was acquired with a Siemens-product point-resolved spectroscopy pulse-sequence (repetition time/echo time $=2,000 / 30$ milliseconds, 96 repetitions, voxel dimensions $15 \times 15 \times 15$ $\mathrm{mm}^{3}$, runtime $\left.3: 20\right)$. Each run was immediately followed by an identical second-run (eight repetitions only) with water-suppression turned off. Spectra were fit offline with the LCModel commercial software package. ${ }^{79}$ Among other common proton metabolites, the combined levels of creatine (Cr) and phosphocreatine (PCr) were assayed. (These two metabolites are typically measured together, since their peaks in the MR spectrum are overlapping and poorly segregated and since the two compounds are biochemically coordinated). Metabolite levels were adjusted for the amount of cerebrospinal fluid in the MRS voxel (a potential confound), quantified relative to the unsuppressed water signal (which has a known concentration), and expressed in Institutional Units.

A sample spectrum with $\mathrm{Cr}+\mathrm{PCr}$ peak at a frequency of $3.02 \mathrm{ppm}$ is seen in Figure 2. As shown in Figure 3, pre-CBT $\mathrm{Cr}+\mathrm{PCr}$ in $\mathrm{pACC}$ correlated significantly and negatively with Y-BOCS at follow-up ( $r=-0.54, P<0.05$, Spearman); that is, patients with higher $\mathrm{Cr}+\mathrm{PCr}$ before $\mathrm{CBT}$ had less severe OCD symptoms at follow-up; patients with lower $\mathrm{Cr}+\mathrm{PCr}$ had more severe symptoms. A significant negative correlation was also seen between $\mathrm{Cr}+\mathrm{PCr}$ and $\mathrm{OCD}$ symptom severity expressed by the Obsessive-Compulsive Index $(r=-0.63, P<0.01)$. Higher pre-CBT $\mathrm{Cr}+\mathrm{PCr}$ also predicted lower comorbid depressive $(r=-0.71, P<0.001)$ and anxious $(r=-0.68, P<0.001)$ symptoms at follow-up, as evaluated by the Montgomery-Åsberg Depression Rating Scale and the Hamilton Anxiety Rating Scale. Finally, pre-CBT $\mathrm{Cr}+\mathrm{PCr}$ correlated negatively with the Sheehan Disability Score at follow-up $(r=-0.75, P<0.001$, not shown), meaning that greater disability was observed for patients with lower pretreatment $\mathrm{Cr}+\mathrm{PCr}$. Thus, low initial $\mathrm{Cr}+\mathrm{PCr}$ in the $\mathrm{pACC}$ brain region presaged poor outcome on multiple clinical dimensions at least at one timepoint in the first post-treatment year. Similar findings (not shown) pertained for other MRS metabolites related to energy metabolism, such as choline compounds (Cho).

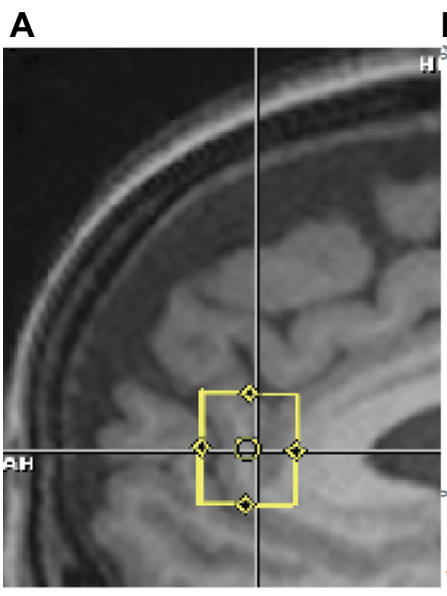

B

Figure 2 Sagittal MRI of pregenual anterior cingulate and its proton magnetic resonance spectrum.

(A) Sagittal TI-weighted MRI of the human brain showing location of $15 \times 15 \times 15 \mathrm{~mm}^{3}$ proton magnetic resonance spectroscopy (MRS) acquisition voxel (yellow square) in the pregenual anterior cingulate cortex (pACC). (B) Sample raw (jagged gray), baseline (light gray), and fit (solid red) MR spectra acquired from the pACC. The MR spectrum is a plot of the intensity of radiofrequency signal (ordinate axis) vs signal frequency (abscissa) in parts-per-million (ppm). The size (area under the curve) of each peak in the spectrum is proportional to a different biochemical species (metabolite) or small family of chemically related metabolites in the MRS voxel. The peak marked "Cr+PCr" at $3.02 \mathrm{ppm}$ represents the sum of the energetic neurometabolites creatine and phosphocreatine. In our OCD pilot sample, pretreatment $\mathrm{Cr}+\mathrm{PCr}$ level in $\mathrm{pACC}$ was predictive of OCD core and other symptoms at post-treatment follow-up.

Abbreviations: $\mathrm{Cr}$, creatine; OCD, obsessive-compulsive disorder; MR, magnetic resonance; MRI, magnetic resonance imaging; PCr, phosphocreatine. 

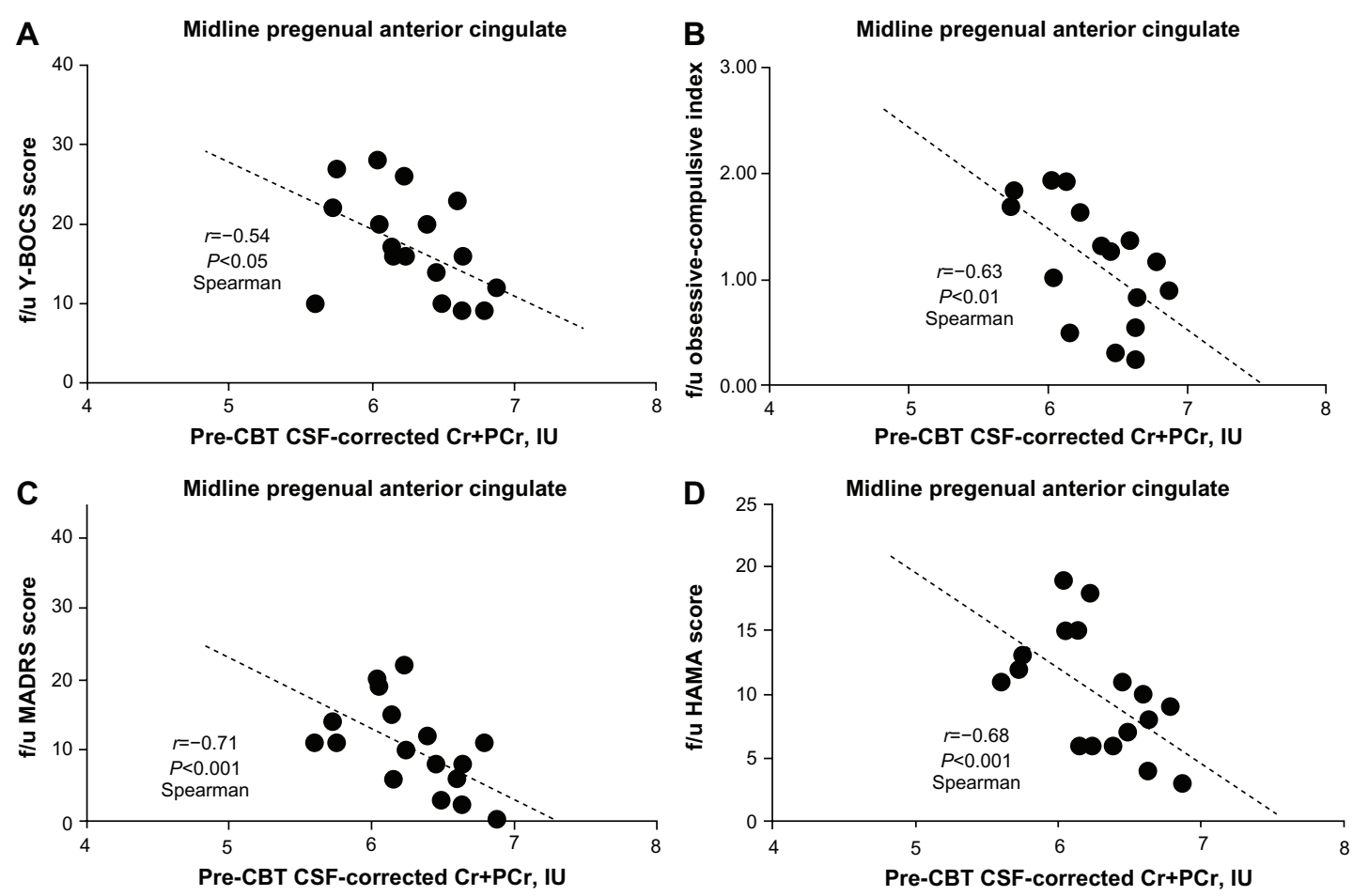

Figure 3 Post-treatment ( $\mathrm{I}-12$ months) follow-up (f/u) clinical scores in a sample of 19 adult OCD patients (filled circles) who received 4 weeks of intensive daily CBT. Notes: (A and B) Scores assayed core OCD symptoms on the Yale-Brown Obsessive Compulsive Scale (Y-BOCS, A) and Obsessive-Compulsive Inventory (OCI, B). (C and D) Scores assayed depressive symptoms on the Montgomery-Åsberg Depression Rating Scale (MADRS, C) and anxious symptoms on the Hamilton Anxiety Rating Scale (HAMA, D). All scores are plotted as a function of the combined level (corrected for cerebrospinal fluid [CSF] content of the acquisition volume) of the energetic neurometabolites creatine $(\mathrm{Cr})$ and phosphocreatine $(\mathrm{PCr})$ in the midline (left + right) pregenual anterior cingulate cortex (pACC), a brain region linked to positive affect, among other functions. Levels were acquired with proton magnetic resonance spectroscopy (MRS) at most I week prior to CBT. Higher pretreatment levels of Cr+PCr were significantly correlated (Spearman) with lower OCD, depressive, and anxious symptoms across the sample. Thus, elevated energetic metabolism may dispose toward retention of response to CBT. This suggests that brain imaging may be helpful in predicting long-term outcomes in OCD.

Abbreviations: CBT, cognitive-behavioral therapy; CSF, cerebrospinal fluid; IU, Institutional Units; OCD, obsessive-compulsive disorder

The precise physiological functions of the standard MRS neurometabolites are not firmly established, but we do have reasonable educated guesses. $\mathrm{Cr}$ and $\mathrm{PCr}$ together enable the short-term storage and release of cell-metabolic energy through the interchange of adenosine triphosphate and adenosine diphosphate.$^{80} \mathrm{It}$ is reasonable to think that higher combined $\mathrm{Cr}+\mathrm{PCr}$, measured by proton MRS, corresponds to greater buffering capacity, and thus greater ability to handle high energy throughout in the pACC. The pACC is implicated, among other functions, in regulation of positive emotional affect. ${ }^{81,82}$ It forms, for example, brain-reward circuits with the nucleus accumbens and thalamus. ${ }^{83}$ A possible predisposing role for malfunctioning positive valence systems of the brain in OCD has been postulated. ${ }^{84}$ The dominant day-to-day affective tone in OCD is strongly negative, marked mainly by fear, worry, anxiety, perhaps at times by panic, but also, to degrees, by guilt, shame, regret and other ill feelings. In an, again speculative, interpretation of our findings, elevated $\mathrm{Cr}+\mathrm{PCr}$ may reflect greater ability of the patient to experience and to be motivated by positive emotions and thereby to acclimate to life after $\mathrm{CBT}$. Lower $\mathrm{Cr}+\mathrm{PCr}$ in $\mathrm{pACC}$ may reflect less capacity to reorient thinking and behavior around positive motivations and thus may predispose a patient to slip back into pre-established OCD habits, ie, to relapse. In any case, these findings suggest an ability of regional MRS neurometabolites to predict post-CBT symptom severity and everyday functioning in OCD.

\section{A neuroimaging research program to predict post-CBT outcome in OCD}

Improved prediction of $\mathrm{CBT}$ response in $\mathrm{OCD}$, particularly of long-term outcomes, could improve access to CBT by lowering the investment risks in terms of therapists' and patients' time and effort and reimbursement costs for thirdparty payers. One possible pathway to seek these goals is the following. Although there is no guarantee that these goals can be achieved, building on the above-described research, this program outlines plausibly attainable next steps, not for the far, but for the immediate future. The first step is to validate predictive imaging results, such as our sample rsfMRI and MRS findings above, in larger patient groups, and using more 
regular and extended post-treatment follow-up intervals. We point out that the rsfMRI and MRS procedures described can each be carried out in approximately 10 minutes on conventional MRI scanners. These scanners are already widely available at hospitals, medical research institutes, and local imaging centers in many areas. The next step is to identify endpoints (if such exist) that yield a cleaner and larger separation between relapsers and patients maintaining good response. Ie, the above findings are striking but, ideally and for clinical purposes, we desire a larger gap between subject groups with minimal overlap to reduce false positives and false negatives. This can be approached in at least two ways. The first is to test further metrics and combinations of metrics. For example, perhaps a non-path length or clustering coefficient-dependent graph theory metric, such as the participation coefficient, will perform better than small-worldness in discriminating groups. Or two graph theory metrics combined or two MRS metabolite levels or graph theory plus MRS. Collecting data from larger patient samples will open the field for this type of analytic experimentation and identification of optimum imaging endpoints. The second is that stratification (eg, by sex, age, severity, age-of-onset, and dimensional profile) of patient populations may lead to sharper distinctions between outcome groups on imaging indices. This will be enabled by studying larger overall samples. Once a clear and reliable predictive separation is established, as mentioned, the third step is to attain cross-scanner compatibility (eg, between Siemens and GE scanners) of the imaging endpoints. Thereafter, the path would not be far to clinical applicability. In patients free of MRI contraindications (eg, pacemakers and certain other implants), the rsfMRI and MRS scans described above can be acquired in 15-30 minutes using pulse sequences delivered with most scanners. The rsfMRI and MRS post-processing software we used above is available in streamlined commercial or freely downloadable open-source packages with large and active user support communities. The procedures can be readily automated for operation by non-specialist clinicians. Ultimately, clinicians could use the predictions obtained in clinical decision-making. For example, those with high likelihood of relapse or worsening of symptoms after CBT could be diverted to other forms of treatment instead such as medications or newer non-invasive and (for severe, refractory cases) invasive brain stimulation techniques (which, in kind, would benefit from similar lines of predictive research).

We emphasize that putative imaging predictors of CBT response represent only a partial and potential solution to the issue of access to CBT for OCD. Other solutions, such as training more therapists and developing alternative CBT modalities, should continue to be pursued. And again, no patient who wants CBT should be denied access to it simply because imaging indicators foresee a poor response. The main purpose of imaging predictors of response should be to help patients and clinicians better choose among their options. Moreover, current common practice of first exhausting safer treatments, including CBT, before resorting to invasive therapies, such as deep brain stimulation or surgical resections, should remain even if highly accurate imaging predictors are established.

\section{Conclusion}

OCD is a widespread and often highly debilitating psychiatric condition, yet effective treatments are highly under-accessed. CBT is a first-line therapy for OCD, but is particularly underused. Many patients may prefer SRI medication because they are intimidated by the psychological challenges of CBT and/or reluctant to invest the work involved. Other barriers to access include the shortage of therapists trained in CBT, as well as the expense of CBT and difficulties with insurance coverage for the same. Group-CBT and Internetbased CBT are emerging as effective practical responses to cost and therapist-shortage issues. A further innovative response might be improved prediction - perhaps with the aid of non-invasive neuroimaging - of long-term outcomes after CBT. In addition to lowering patient burden, more reliable outcome prediction would better justify cost reimbursement and would enable superior allocation of clinical resources. Pilot data from our laboratory were presented demonstrating that pretreatment endpoints derived from whole-brain rsfMRI analyzed using graph theory and MRS of the pACC brain region correlate with Y-BOCS and other clinical scores in post-CBT follow-up.

\section{Acknowledgments}

The authors wish to thank Karron Maidment, RN, MFT, and Lindsey Bergman, $\mathrm{PhD}$, for informative discussion. This research is supported by NIMH awards R01MH085900 (JON/ JF) and R01MH081864 (JON and John Piacentini, PhD).

\section{Disclosure}

JDF is on the Scientific and Clinical Advisory Board of the International Obsessive-Compulsive Disorder Foundation. The authors report no other conflicts of interest in this work.

\section{References}

1. Bystritsky A, Munford PR, Rosen RM, et al. A preliminary study of partial hospital management of severe obsessive compulsive disorder. Psychiatr Serv. 1996;47(2):170-174. 
2. Calvocoressi L, McDougle CI, Wasylink S, Goodman WK, Trufan SJ, Price LH. Inpatient treatment of patients with severe obsessive-compulsive disorder. Hosp Community Psychiatry. 1993;44(12):1150-1154.

3. Eddy KT, Dutra, Bradley R, Westen D. A multidimensional metaanalysis of psychotherapy and pharmacotherapy for obsessivecompulsive disorder. Clin Psych Rev. 2004;24:1011-1030.

4. Abramowitz JS. The psychological treatment of obsessive-compulsive disorder. Can J Psychiatry. 2006;51:407-416.

5. Simpson HB, Huppert JD, Petkova E, Foa EB, Liebowitz MR. Response versus remission in obsessive-compulsive disorder. $J$ Clin Psychiatry. 2006;67(2):269-276.

6. Eisen JL, Goodman WK, Keller MB, et al. Patterns of remission and relapse in obsessive-compulsive disorder: a 2-year prospective study. J Clin Psychiatry. 1999;60(5):346-352.

7. Foa EB, Kozak MJ. Psychological treatment for obsessive-compulsive disorder. In: Mavissakalian M, Prien R, editors. Long-Term Treatments of Anxiety Disorders. Washington, DC: American Psychiatric Press, Inc.; 1996:285-309.

8. Maina G, Albert U, Bogetto F. Relapses after discontinuation of drug associated with increased resistance to treatment in obsessivecompulsive disorder. Int Clin Psychopharm. 2001;16(1):33-38.

9. Ravizza L, Barzega G, Bellino S, Bogetto F, Maina G. Drug treatment of obsessive-compulsive disorder (OCD): long-term trial with clomipramine and selective serotonin reuptake inhibitors (SSRIs) Psychopharm Bull. 1996;32(1):167-173.

10. Romano S, Goodman W, Tamura R, Gonzales J, The Collaborative Research Group. Long-term treatment of obsessive-compulsive disorder after an acute response: a comparison of fluoxetine versus placebo. J Clin Psychopharm. 2001;21(1):46-52.

11. Simpson HB, Liebowitz MR, Foa EB, et al. Post-treatment effects of exposure therapy and clomipramine in obsessive compulsive disorder. Depress Anxiety. 2004;19(4):225-233.

12. Simpson HB, Franklin ME, Cheng J, Foa EB, Liebowitz MR. Standard criteria for relapse are needed in obsessive-compulsive disorder Depress Anxiety. 2005;21(1):1-8.

13. Koran LM, Hanna GL, Hollander E, Nestadt G, Simpson HB, American Psychiatric Association. Practice guideline for the treatment of patients with obsessive-compulsive disorder. Am J Psychiatry. 2007; 164(7 Suppl):5-53.

14. Marques L, LeBlanc NJ, Weingarden HM, Timpano KR, Jenike M, Wilhelm S. Barriers to treatment and service utilization in an Internet sample of individuals with obsessive-compulsive symptoms. Depress Anxiety. 2010;27:470-475

15. Goodwin R, Koenen KC, Hellman F, Guardino M, Struening E. Helpseeking and access to mental health treatment for obsessivecompulsive disorder. Acta Psychiatr Scand. 2002;106:143-149.

16. Kohn DA, Saxena S, Levav I, Saraceno B. The treatment gap in mental health care. Bull World Health Organ. 2004;82:858-866.

17. Schwartz C, Schlegl S, Kuelz AK, Voderholzer U. Treatment-seeking in OCD community cases and psychological treatment actually provided to treatment-seeking patients: a systematic review. J Obsessive Compuls Relat Disord. 2013;2448-2456.

18. Blanco C, Olfson M, Stein DJ, Simpson HB, Gameroff MJ, Narrow WH. Treatment of obsessive-compulsive disorder by US psychiatrists. J Clin Psychiatry. 2006;67:946-951.

19. Torres AR, Prince MJ, Bebbington PE, et al. Treatment seeking by individuals with obsessive-compulsive disorder from the British psychiatric morbidity survey of 2000. Psychiatr Serv. 2007;58:977-982.

20. American Psychiatric Association. Diagnostic and Statistical Manual of Mental Disorders: DSM-5. Washington, DC: American Psychiatric Association; 2013.

21. Taylor CB, Chang VY. Issues in the dissemination of cognitive-behavior therapy. Nord J Psychiatry. 2008;62:37-44.

22. Larsson BPM, Kaldo V, Broberg AG. Similarities and differences between practitioners of psychotherapy in Sweden: a comparison of attitudes between psychodynamic, cognitive, cognitive-behavioral, and integrative therapists. J Psychother Integr. 2009;19(1):34-66.
23. Mataix-Cols D, Marks IM. Self-help with minimal therapist contact for obsessive-compulsive disorder: a review. Eur Psychiatry. 2006;21: $75-80$.

24. Shapiro DA, Cavanagh K, Lomas H. Geographic inequity in the availability of cognitive behavioural therapy in England and Wales. Behav Cog Psychother. 2003;31:185-192.

25. Valderhaug R, Götestam K, Larsson B. Clinicians' views on management of obsessive-compulsive disorders in children and adolescents. Nord J Psychiatry. 2004;58:125-132.

26. Anderson RA, Rees CS. Group versus individual cognitive-behavioural treatment for obsessive-compulsive disorder: a controlled trial. Behav Res Ther. 2007;45:123-137.

27. Belotto-Silva C, Diniz JB, Malavazzi DM, et al. Group cognitivebehavioral therapy versus selective serotonin reuptake inhibitors for obsessive-compulsive disorder: a practical clinical trial. J Anxiety Disord. 2012;26:25-31.

28. Bouvard M, Milliery M, Cottraux J. Un programme de thérapie cognitive et comportementale en groupe dans le trouble obsessionnel compulsif: résultats préliminaires. Encéphale. 2002;28(5 Pt 1):439-446.

29. Braga DT, Gus Manfro G, Niederauer K, Cordiolo AV. Full remission and relapse of obsessive-compulsive symptoms after cognitivebehavioral group therapy: a two-year follow-up. Rev Bras Psiquiatria. 2010;32(2):164-168.

30. Farrell L, Waters A, Milliner E, Ollendick T. Comorbidity and treatment response in pediatric OCD: a pilot study of group cognitive-behavioral treatment. Psychiatry Res. 2012;199(2):115-123.

31. Andersson E, Enander J, Andrén P, et al. Internet-based cognitive behaviour therapy for obsessive-compulsive disorder: a randomized controlled trial. Psychol Med. 2012;42:2193-2203.

32. Andersson E, Ljótsson B, Hedman E, et al. Internet-based cognitive behavior therapy for obsessive compulsive disorder: a pilot study. $B M C$ Psychiatry. 2011;11:125-135.

33. Klein B, Meyer D, Austin DW, Kyrios M. Anxiety Online - A virtual clinic: preliminary outcomes following completion of five fully automated treatment programs for anxiety disorders and symptoms. $J$ Med Internet Res. 2011;13(4):e89.

34. Lenhard F, Vigerland S, Andersson E, et al. Internet-delivered cognitive behavior therapy for adolescents with obsessive-compulsive disorder: an open trial. PLoS One. 2014;9(6):e100773.

35. Storch EA, Caporino NE, Morgan JR, et al. Preliminary investigation of web-camera delivered cognitive-behavioral therapy for youth with obsessive-compulsive disorder. Psychiatry Res. 2011;189: 407-412.

36. Turner CM, Matiax-Cols D, Lovell K, et al. Telephone cognitivebehavioral therapy for adolescents with obsessive-compulsive disorder: a randomized controlled non-inferiority trial. J Am Acad Child Adol Psychiatry. 2014;53(12):1298-1307.

37. Hiss H, Foa EB, Kozak MJ. Relapse prevention program for treatment of obsessive-compulsive disorder. J Consult Clin Psych. 1994;62(4): 801-808.

38. Kirk JW. Behavioural treatment of obsessional-compulsive patients in routine clinical practice. Behav Res Ther. 1983;21(1):57-62.

39. Meyer V. Modification of expectations in cases with obsessional rituals. Behav Res Ther. 1966;4(4):273-280.

40. Neziroglu F, Henricksen J, Yaryura-Tobias JA. Psychotherapy of obsessive-compulsive disorder and spectrum: established facts and advances, 1995-2005. Psychiatr Clin North Am. 2006;29(2):585-604.

41. O’Sullivan G, Noshirvani H, Marks I, Monteiro W, Lellott P. Six-year follow-up after exposure and clomipramine therapy for obsessive compulsive disorder. J Clin Psychiatry. 1991;52(4):150-155.

42. Riggs D, Foa EB. Obsessive compulsive disorder. In: Barlow DH, editor. Clinical Handbook of Psychological Disorders: A Step-byStep Treatment Manual. 2nd ed. New York: Guilford Press; 1993: 189-239.

43. Steketee G, Foa EB, Grayson JB. Recent advances in the behavioral treatment of obsessive-compulsives. Arch Gen Psychiatry. 1982;39(12): 1365-1371. 
44. Pallanti S, Hollander E, Bienstock C, et al. Treatment non-response in OCD: methodological issues and operational definitions. Int $J$ Neuropsychopharm. 2002;5(2):181-191.

45. Koran LM, Hackett E, Rubin A, Wolkow R, Robinson D. Efficacy of sertraline in the long-term treatment of obsessive-compulsive disorder. Am J Psychiatry. 2002;159(1):88-95.

46. Hollander E, Paroxetine OCD Study Group, Allen A, et al. Acute and long-term treatment and prevention of relapse of obsessive-compulsive disorder with paroxetine. J Clin Psychiatry. 2003;64(9):1113-1121.

47. Hollander E, Stein DJ, Fineberg NA, Marteau F, Legault M. Quality of life outcomes in patients with obsessive-compulsive disorder: Relationship to treatment response and symptom relapse. J Clin Psychiatry. 2010;71(6):784-792.

48. Fineberg NA, Tonnoir B, Lemming O, Stein DJ. Escitalopram prevents relapse of obsessive-compulsive disorder. Eur Neuropsychopharm. 2007;17(6-7):430-439.

49. Foa EB, Liebowitz MR, Kozak MJ, et al. Randomized, placebocontrolled trial of exposure and ritual prevention, clomipramine, and their combination in the treatment of obsessive-compulsive disorder. Am J Psychiatry. 2005;162(1):151-161.

50. Keeley ML, Storch EA, Merlo LJ, Gefken GR. Clinical predictors of response to cognitive-behavioral therapy for obsessive-compulsive disorder. Clin Psychol Rev. 2008;28:118-130.

51. Raffin AL, Ferrão YA, Pasquoto de Souza F, Cordioli AV. Fatores preditores de resultados no tratamento do transtorno obsessivo-compulsivo com as terapias comportamental e cognitivo-comportamental: uma revisão sistemática. Rev Psiquiatria do Rio Grande do Sul. 2008;30(1 Suppl): $1-20$.

52. Knopp J, Knowles S, Bee P, Lovell K, Bower P. A systematic review of predictors and moderators of response to psychological therapies in OCD: do we have enough empirical evidence to target treatment? Clin Psych Rev. 2013;33:1067-1081.

53. Eisen JL, Pinto A, Mancebo MC, Dyck IR, Orlando ME, Rasmussen SA. A 2-year prospective follow-up study of the course of obsessivecompulsive disorder. J Clin Psychiatry. 2010;71(8):1033-1039.

54. Lewin AB, Wu MS, McGuire JF, Storch EA. Cognitive behavior therapy for obsessive-compulsive and related disorders. Psychiatr Clin North Am. 2014;37(3):415-445.

55. Bloch MH, Green C, Kichuk SA, et al. Long-term outcome in adults with obsessive-compulsive disorder. Depress Anxiety. 2013;30(8):716-722.

56. Eisen JL, Sibrava NJ, Boisseau CL, et al. Five-year course of obsessivecompulsive disorder: Predictors of remission and relapse. J Clin Psychiatry. 2013;74(3):233-239.

57. Fineberg NA, Hengartner MP, Bergbaum C, Gale T, Rössner W, Angst J. Remission of obsessive-compulsive disorders and syndromes; evidence from a prospective community cohort study over 30 years. Int J Psychiatry Clin Pract. 2013;17(3):179-187.

58. Jakubovski E, Diniz JB, Valerio C, et al. Clinical predictors of longterm outcome in obsessive-compulsive disorder. Depress Anxiety. 2013; 30(8):763-772.

59. Rufer M, Hand I, Alsleben H, et al. Long-term course and outcome of obsessive-compulsive patients after cognitive-behavioral therapy in combination with either fluvoxamine or placebo: a 7-year follow-up of a randomized double-blind trial. Eur Arch Psychiatry Clin Neurosci. 2005;255(2):121-128.

60. Stewart SE, Geller DA, Jenike M, et al. Long-term outcome of pediatric obsessive-compulsive disorder: a meta-analysis and qualitative review of the literature. Acta Psychiatr Scand. 2004;110(1):4-13.

61. Farris SG, McLean CP, Van Meter P, Simpson HB, Foa EB. Comorbidity and treatment response in pediatric OCD: a pilot study of group cognitivebehavioral treatment. J Clin Psychiatry. 2013;74(7):685-690.

62. Gerber AJ, Peterson BS. What is an image? J Am Acad Child Adolesc Psychiatry. 2008;47(3):245-248.
63. Maia TV, Cooney RE, Peterson BS. The neural bases of obsessivecompulsive disorder in children and adults. Dev Psychopathol. 2008;20: 1251-1283.

64. Menzies L, Chamberlain SR, Laird AR, Thelen SM, Sahakian BJ, Bullmore ET. Integrating evidence from neuroimaging and neuropsychological studies of obsessive-compulsive disorder: the orbitofrontostriatal model revisited. Neurosci Biobehav Rev. 2008;32:525-549.

65. Cole MW, Bassett DS, Power JD, Braver TS, Petersen SE. Intrinsic and task-evoked network architectures of the human brain. Neuron. 2014; $83: 238-251$

66. Doidge N. The Brain that Changes Itself. New York: Viking Press; 2007.

67. Stern ER, Fitzgerald KD, Welsh RC, Abelson JL, Taylor SF. Resting-state functional connectivity between fronto-parietal and default mode networks in obsessive-compulsive disorder. PLoS One. 2012;7(5):e36356.

68. van den Heuvel OA, Veltman DJ, Groenewegen HJ, et al. Frontal-striatal dysfunction during planning in obsessive-compulsive disorder. Arch Gen Psychiatry. 2005;62:301-310.

69. Brennan BP, Rauch SL, Jensen JE, Pope HG Jr. A critical review of magnetic resonance spectroscopy studies of obsessive-compulsive disorder. Biol Psychiatry. 2013;73(1):24-31.

70. O’Neill J, Piacentini JC, Chang S, et al. MRSI correlates of cognitivebehavioral therapy in pediatric obsessive-compulsive disorder. Prog Neuropsychopharmacol Biol Psychiatry. 2012;36:161-168.

71. Saxena S, Gorbis E, O’Neill J, et al. Rapid effects of brief intensive cognitive-behavioral therapy on brain glucose metabolism in obsessivecompulsive disorder. Mol Psychiatry. 2009a;14(2):197-205.

72. Saxena S, O'Neill J, Rauch S. The role of cingulate cortex dysfunction in obsessive-compulsive disorder. In: Vogt BA, editor. Cingulate Neurobiology and Disease. New York: Oxford University Press; 2009b:587-617.

73. O’Neill J, Gorbis E, Feusner JD, et al. Effects of intensive cognitivebehavioral therapy on cingulate neurochemistry in obsessive-compulsive disorder. J Psychiatry Res. 2013;47(4):494-504.

74. Feusner J, Moody T, Lai TM, et al. Brain connectivity and prediction of relapse after cognitive-behavioral therapy in obsessive-compulsive disorder. Front Psychiatry. 2015;6:74.

75. Bullmore E, Sporns O. Complex brain networks: graph theoretical analysis of structural and functional systems. Nat Rev Neurosci. 2009; 10(3):186-198

76. Watts DJ, Strogatz SH. Collective dynamics of 'small-world' networks. Nature. 1998;393(6684):440-442.

77. Zhang T, Wang J, Yang Y, et al. Abnormal small-world architecture of top-down control networks in obsessive-compulsive disorder. J Psychiatry Neurosci. 2011;36(1):23-31.

78. Shin DJ, Wi HJ, He Y, et al. The effects of pharmacological treatment on functional brain connectome in obsessive-compulsive disorder. Biol Psychiatry. 2014;75(8):606-614.

79. Provencher SW. Automatic quantitation of localized in vivo ${ }^{1} \mathrm{H}$ spectra with LCModel. NMR Biomed. 2001;14(4):260-264.

80. Erecinska M, Silver IA. ATP and brain function. J Cereb Blood Flow Metab. 1989;9(1):2-19.

81. Vogt BA. Pain and emotion interactions in subregions of the cingulate gyrus. Nat Rev Neurosci. 2005;6(7):533-544.

82. Vogt BA. Regions and subregions of the cingulate gyrus. In: Vogt BA, editor. Cingulate Neurobiology and Disease. New York: Oxford University Press; 2009.

83. Berridge KC, Kringelbach ML. Affective neuroscience of pleasure: reward in humans and animals. Psychopharmacolgy (Berl). 2008;199:457-480.

84. Aouizerate B, Guehl D, Cuny E, et al. Pathophysiology of obsessivecompulsive disorder: a necessary link between phenomenology, neuropsychology, imagery and physiology. Prog Neurobiol. 2004;72:195-221. 


\section{Publish your work in this journal}

Psychology Research and Behavior Management is an international, peerreviewed, open access journal focusing on the science of psychology and its application in behavior management to develop improved outcomes in the clinical, educational, sports and business arenas. Specific topics covered include: Neuroscience, memory \& decision making; Behavior modification \& management; Clinical applications; Business \& sports performance management; Social and developmental studies; Animal studies. The manuscript management system is completely online and includes a quick and fair peer-review system. Visit http://www.dovepress. com/testimonials.php to read real quotes from published authors.

Submit your manuscript here: http://www.dovepress.com/psychology-research-and-behavior-management-journal 